\title{
Role of microRNAs in peripheral artery disease (Review)
}

\author{
XIANGYU ZHOU ${ }^{1,2}$, PING YUAN ${ }^{3}$ and YANZHENG HE ${ }^{1}$ \\ ${ }^{1}$ Department of Vascular Surgery, Affiliated Hospital of Luzhou Medical College, Luzhou, Sichuan 646000, P.R. China; \\ ${ }^{2}$ Department of Pharmacology, Rush University Medical Center, Chicago, IL 60616, USA; \\ ${ }^{3}$ Department of Cerebral Vascular Medicine, Affiliated Hospital of Luzhou Medical College, \\ Luzhou, Sichun 646000, P.R. China
}

Received February 16, 2012; Accepted July 2, 2012

DOI: $10.3892 / \mathrm{mmr} .2012 .978$

\begin{abstract}
Peripheral arterial disease (PAD) involves a general vascular problem of diffuse atherosclerosis. The key pathological process is characterized by the aberrant proliferation of vascular smooth muscle cells and the formation of neointimal lesions. The molecular mechanisms involved in the regulation of the occurrence and development of PAD remain unclear. microRNAs (miRNAs) are highly conserved 20-25 nt-long non-coding RNAs that negatively regulate gene expression. Recent evidence has demonstrated that specific miRNAs are involved in the pathological processes of PAD, and these miRNAs are found to be critical modulators of vascular cell functions, including cell differentiation, contraction, migration, proliferation and apoptosis. This review summarizes findings of studies regarding the roles of specific miRNAs in PAD.
\end{abstract}

\section{Contents}

1. Introduction

2. Endothelial cells and miRNAs

3. Vascular smooth muscle cells and miRNAs

4. Diabetes mellitus (DM)-associated arteriogenesis and miRNAs

5. miRNAs and peripheral arterial disease (PAD)

6. Conclusion

\section{Introduction}

Peripheral arterial disease (PAD) affects $12-14 \%$ of the general population, and its prevalence increases significantly with age.

Correspondence to: Dr Xiangyu Zhou, Department of Vascular Surgery, Affiliated Hospital of Luzhou Medical College, 25 Taiping Street, Luzhou, Sichuan 646000, P.R. China

E-mail: xiangyuzhou971@gmail.com

Key words: microRNAs, peripheral arterial disease, endothelial cell, vascular smooth muscle cell
PAD is most frequently caused by atherosclerosis (AS), which leads to obstruction of the blood flow in the lower extremities. The most significant risk factors for the development of PAD are diabetes mellitus (DM) and tobacco abuse, although hypertension and hyperlipidemia are also contributing factors $(1,2)$. Despite progress towards the prevention and treatment of PAD, understanding of the pathogenesis remains only at the initial stage. Understanding the cellular and molecular mechanisms that lead to the development of PAD is critical for identifying strategies to limit disease progression before it has clinical consequences.

Recent studies (3) have paid increasing attention to the cellular and molecular mechanisms of PAD, in which microRNAs (miRNAs) have been the focus. miRNAs are an emerging class of highly conserved, non-coding small RNAs (20-25 nucleotides) that regulate gene expression at the post-transcriptional level, inhibiting the translation of protein from messenger RNA (mRNA) by promoting the degradation of mRNA. miRNAs are key regulators of numerous events, including the balance between cell proliferation and differentiation during tumorigenesis and organ development. At present, more than 600 human miRNAs have been identified, and it has been proposed that they regulate over $50 \%$ of human protein-coding genes $(4,5)$. Significantly, one miRNA is able to regulate the expression of multiple genes due to its ability to bind to its mRNA targets as either an imperfect or perfect complement. Thus, one miRNA is as functionally important as a transcription factor. As a group, miRNAs may directly regulate at least $30 \%$ of the genes in a cell and therefore are involved in the regulation of all major functions (6).

Over the last few years, there has been an increase in miRNA research due to the identified significant roles of these non-coding RNAs in cardiovascular pathophysiology, including arteriosclerosis, angiogenesis and intima hyperplasia. Basic and clinical studies have suggested that miRNAs are important regulators of vascular cell differentiation, growth, proliferation and apoptosis (7-9). However, their biological roles in PAD have only been elucidated recently. Several studies, including our research group, have demonstrated that miRNAs are important in PAD and PAD-associated complications. In the present review, basic and clinical research regarding miRNAs in PAD is summarized and a perspective for this new frontier of peripheral arterial ischemic disease research is provided. 


\section{Endothelial cells and miRNAs}

It is estimated that an adult human has approximately $1-10 \times 10^{12}$ endothelial cells (ECs) lining the inside of blood vessels. Among these cells, only approximately $0.01 \%$ are estimated to be present in the cell cycle at any given time (10). ECs are major regulators of vascular health. During certain physiological events, including wound healing and oxidative stress, alterations in hemodynamic forces or pathological stimuli, such as inflammation or AS, cause ECs to assume a phenotype that predisposes to proatherogenic alterations (11). Therefore, evaluation of endothelial functions may provide insights into the mechanisms underlying the initiation, progression and clinical manifestations of PAD. A number of previous studies have verified the particular role of miRNAs in regulating the function of vascular ECs.

Human umbilical vein endothelial cells (HUVECs) are a valuable in vitro model of angiogenesis due to their ability to form capillary-like structures in response to appropriate stimuli (12). The miRNA signature of HUVECs has been recently determined, and several highly expressed miRNAs have been found to have angiogenic growth factor receptors as their putative targets (13). Among these, the following miRNAs play important roles.

$m i R-21$. miR-21, a negative modulator of angiogenesis, has been demonstrated to be highly expressed in ECs. A study by Sabatel et al demonstrated that miR-21 overexpression reduced EC proliferation, migration and the ability of these cells to form tubes, while the inhibiton of miR-21 using a LNA-anti-miR led to opposite effects (14). Other studies (15) have demonstrated that the overexpression of miR-21 also led to a reduction in the organization of actin into stress fibers, which may explain the decrease in cell migration. Results from another study demonstrated that miRNA-21 exhibits an antiangiogenic function by targeting RhoB expression in ECs (16). Findings of other studies have also suggested that mechanical forces associated with blood flow play a role in regulating vascular signaling and gene expression in ECs. miR-21 therefore affects these pathological processes by decreasing apoptosis and activating the nitric oxide (NO) pathway (14). Adhesion of circulating monocytes to vascular ECs is a critical event leading to vascular inflammation and development of AS. In this pathological process, miRNA-21 targets the peroxisome proliferator-activated receptor $\alpha$ $(\operatorname{PPAR} \alpha)$ in an autoregulatory loop to modulate flow-induced endothelial inflammation (17).

miR-126. Adhesion molecules expressed by activated ECs are important in the regulation of leukocyte trafficking to sites of inflammation. Findings by Harris et al (16) demonstrated that the overexpression of miRNAs in EC inhibits vascular cell adhesion molecular 1 expression, which leads to inhibition of leukocyte adherence to ECs (18-20). miR-126 is also involved in cell apoptosis. miR-126 has been found to mediate CXCL12 production, which was enriched in apoptotic bodies, and induced vascular protection by repressing the function of the regulator of $\mathrm{G}$ protein signaling 16 (21). In addition, the beneficial role of miR-126 has been elucidated using the specificity of antagomir-induced silencing of miR-126 in vivo (22). $m i R-221 / 222$. Zhu et al found that miR-221/222 were highly expressed in HUVECs, and overexpression of the miR-221/222 targeted Ets-1, a key endothelial transcription factor of inflammation and tube formation, indirectly regulated the expression of several inflammatory molecules of ECs, which eventually attenuated cell adhesion and migration (23). In addition, miR-221/222 is involved in inflammation-mediated vascular remodeling by regulating the expression of the signal transducer and activator of transcription 5A (STAT5A) (24).

Other miRNAs are also involved in the regulation of ECs. miR-210 overexpression in normoxic EC cells stimulated the formation of capillary-like structures and vascular endothelial growth factor-driven cell migration (25). Oxidized low-density lipoprotein (ox-LDL) mediates EC apoptosis, which plays a critical role in AS. Through in vivo investigations examining the regulation of the expression of Bcl-2, miR-365 was confirmed to modulate ox-LDL-induced EC apoptosis (26). miR-424 promoted post-ischemic angiogenesis in vitro and in mice, which may regulate hypoxia-inducible factor $1 \alpha$ isoforms (27). Oscillatory shear stress (OS) induces the inflammatory response, a critical atherogenic event in which miR-663 has been identified to play a key role in OS-induced inflammatory responses by mediating the expression of the inflammatory gene network in HUVECs (28).

\section{Vascular smooth muscle cells (VSMCs) and miRNAs}

It is well-known that the phenotypic modulation of VSMCs from a differentiated phenotype to a dedifferentiated state, accompanied by accelerated VSMC proliferation, is crucial in the pathogenesis of a variety of proliferative vascular diseases, including AS, hypertension, restenosis after angioplasty or bypass and diabetic vascular complications, including PAD $(29,30)$. Recent studies have established specific miRNAs as significant mediators of the modulation of VSMC phenotype by targeting transcription factors and the cytoskeleton, which act as molecular switches for VSMC differentiation (31).

$m i R-1$. Myocardin is a cardiac- and smooth muscle-specific transcription co-factor that potently activates the expression of downstream target genes, and its overexpression may inhibit the proliferation of SMCs. miR-1 is involved in myocardin-dependent SMC proliferation inhibition (32). In their study, Xie et al suggested that miR-1 plays a critical role in the determination of SMC fate during retinoid acid-induced embryonic stem cell/SMC differentiation in vitro (33).

$m i R-21$. Besides being highly expressed in ECs, miR-21 is a proproliferative and antiapoptotic regulator of VSMCs.

Recently, our group performed a series of in vitro and in vivo studies on miR-21. In vitro we identified that the expression of miR-21 in dedifferentiated VSMC was higher than in fresh isolated differentiated cells. Depletion of miR-21 resulted in decreased cell proliferation and increased cell apoptosis. PTEN and Bcl-2 were involved in miR-21-mediated cellular effects (34). Neointimal lesion formation is the pathological basis of AS, thus in order to investigate the correlation between miR-21 and neointimal lesions, we constructed a rat balloon injury carotid artery model. The results demonstrated 
that miR-21 was aberrantly expressed in the vascular walls. Using the knock-down method it was found that modulating the overexpression of miR-21 had a significant negative effect on neointimal lesion formation (35). Following further investigation, we identified that miR-21 in VSMCs was sensitive to reactive oxygen species (ROS). miR-21 plays a role in $\mathrm{H}_{2} \mathrm{O}_{2}$-mediated gene regulation and the cell injury response through the programmed cell death 4 (PDCD4) and activator protein 1 pathways (36). Propofol is a widely used intravenous anesthetic agent with antioxidant properties. Our group investigated other mechanisms of ROS-induced injury in VSMCs, and results revealed that propofol exacerbates cell injury in VSMCs with increased ROS, partly through miRNA-21 and its target gene, PDCD4 (35).

miR-143/145. miR-143/145 is the most abundant miRNA in normal arteries and is mainly localized in VSMCs (37). It has recently been demonstrated that the downregulation of miRNA-143/145, which are coexpressed from a single promoter, regulates the switch from contractile to synthetic phenotype, allowing SMCs to migrate and proliferate (39). Serum response factor (SRF) and its coactivator, myocardin, play a key role in the control of smooth muscle phenotypes by regulating the expression of cytoskeletal genes. Using in vivo and in vitro studies, Xin et al confirmed that SRF and myocardin regulate a cardiovascular-specific miRNA cluster encoding miR-143/145. miR-143/145 act as integral components of the regulatory network whereby SRF controls cytoskeletal remodeling and phenotypic switching of SMCs during vascular disease (40). Furthermore, Cordes et al demonstrated that miR-145 is able to direct smooth muscle fate, and that miR-143 and -145 function regulates the quiescent versus proliferative phenotype of SMCs, which is able to cooperatively target a network of transcription factors, including Krüppel-like factor 4 (KLF4), myocardin and EIK-1 (41). Platelet-derived growth factor (PDGF) is one of the most potent stimuli for the migration of VSMCs. Studies have shown that PDGF mediates podosome formation in SMCs through the regulation of miR-143/-145 expression via a pathway involving Src and p53.

In a rat model of acute vascular injury, overexpression of miR-143 and -145 decreased neointimal formation, while loss of the miRNAs induced structural modification of the vessel, due to incomplete differentiation of VSMCs (42). Consistent with the above studies, we aimed to determine the exact role of miR-145, and obtained similar results to those of previous studies. Both in cultured rat VSMCs in vitro and in ballooninjured rat carotid arteries in vivo, we demonstrated that the miR-145 target KLF5 and myocardin mediate vascular neointimal growth, which may be a novel phenotypic marker and a novel phenotypic modulator of VSMCs (43).

$m i R-221 / 222$. Studies by other authors and our group have confirmed the role of miR-221/222 in the regulation of VSMCs. Davis et al demonstrated that PDGF signaling, by modulating the expression of miR-221, regulates two critical determinants of the VSMC phenotype, including VSMC gene expression and cell proliferation. miR-221 is transcriptionally induced following PDGF treatment in primary VSMCs, leading to the downregulation of the targets, c-kit and p27 (Kip1), which directly induced cell proliferation $(31,44)$.
Our results support the hypothesis that miR-221/-222 are novel regulators of VSMC proliferation and neointimal hyperplasia. Results of our study showed that miR-221/-222 expression was increased by growth stimulators in cultured VSMCs. Knockdown resulted in a decreased VSCM proliferation in vitro (45). Consistent with results by Davis et al (31), we also identified that as target genes, Kip1 and p57 (kip2) were involved in miR-221/222-mediated effects on VSMC growth. Using an induced rat carotid arterial model after angioplasty, we identified the overexpression of miR-221/222 in injured vascular walls, while in vivo knockdown suppressed VSMC proliferation (46).

Other miRNAs. Other miRNAs are also involved in mediating VSMCs. miR-29b, directly targeting DNMT3b, may mediate epigenetic regulation in AS (47). miR-146a targets KLF4 and plays a role in promoting VSMC proliferation in an in vitro and in vivo model (48). Let-7d regulates proliferation and differentiation, and RAS may also be involved (49).

\section{DM-associated arteriogenesis and miRNAs}

DM is recognized as a major cardiovascular risk factor. Patients suffering from diabetes and PAD are at risk of developing critical limb ischemia and foot ulceration, potentially requiring limb amputation. DM affects arteriogenesis of PAD at a number of levels. miRNAs are involved in the epigenetic regulation of key metabolic, inflammatory and antiangiogenic pathways in DM and may contribute to common disease complications. miR-126 is the most abundant miRNA in endothelial apoptotic bodies. Shedding of miR-126 from ECs has been demonstrated to regulate VEGF responsiveness and to confer vascular protection. miR-126 may contribute to VEGF resistance and endothelial dysfunction in DM (50). miR-208 is involved in insulin-induced VSMC proliferation via the downregulation of its potential target, p21, a key member of the cyclin-dependent kinase inhibitory protein family $(51,52)$. miR-221 regulates a high glucose-induced endothelial dysfunction. Under hyperglycemic conditions, miR-221 triggers the inhibition of c-kit and impairment of HUVECs migration (53). In high glucose- and AGE-mediated vascular damage, miR-221/-222 targeting Kip1 and Kip2 regulate vascular cell proliferation (54). Wang et al revealed that miR-320 is associated with impaired angiogenesis in DM. Transfection of miR-320 inhibitor may be a therapeutic approach for the treatment of impaired DM-associated angiogenesis (55).

\section{5. miRNAs and PAD}

Ischemic complications are the leading cause of morbidity and mortality in PAD patients. An improved understanding of the molecular mechanisms of limb ischemia is required to improve therapeutic options. miR-92a has been identified as an endogenous repressor of the angiogenic program. Overexpression of miR-92a blocks angiogenesis and vessel formation. Based on this, using a mouse hind-limb ischemia model, Bonauer et al identified that ischemic injury significantly increased the expression of miR-92a, and injection of antagomir-92a reduced necrosis, improved perfusion and caused functional recovery of ischemic limbs. These results suggest that in the setting of 
ischemic disease, miRNA-92a, targeted by several proangiogenic proteins including subunit $\alpha 5$, controls angiogenesis and the functional recovery of ischemic tissues (56). Grundmann et al found that miRNA-100 was significantly downregulated following the induction of hind-limb ischemia in mice. It was demonstrated that miR-100 has an antiangiogenic function and represses the mammalian target of rapamycin (mTOR) signaling in vitro, which modulates proliferation and tube formation (57). miR-322/-424 is a critical mediator of oxygendependent changes in ECs and is physiologically upregulated in tissues undergoing vascular remodeling and angiogenesis. Hypoxia differentially increased miRNA-424 levels in ECs and miR-424 targeted cullin 2 (CUL2), which plays an important physiological role in post-ischemic vascular remodeling and angiogenesis (27).

In addition to the above basic investigations concerning PAD, there is now a focus on clinical studies. Arteriosclerosis obliterans (ASO) is a type of PAD. Wang et al (58) investigated the role of miR-21 in human arteries and VSMCs with ASO. The results of those authors suggested that miR-21 is capable of regulating ASMC function by targeting tropomyosin 1, and the hypoxia inducible factor- $1 \alpha / \mathrm{miR}-21 /$ tropomyosin 1 pathway may play a critical role in the pathogenesis of ASO (58). Specific signatures of miRNAs may be obtained from total serum/plasma or tissues and are able to be used in the identification of biomarkers for the diagnosis, prognosis or even the etiology of a disease (59). Currently, miRNAs have become a hot topic as biomarkers and therapeutic targets for cardiovascular disease (60). Caporali et al (59) revealed that miR-503 was highly expressed in ischemic muscle specimens and the plasma of diabetic patients with critical limb ischemia. Their study provided evidence for a role of miR-503 in DM-induced endothelial defects contributing to impaired postischemic angiogenesis, and also demonstrated that the overexpression of miR-503 inhibited EC proliferation, migration and network formation, and reduced VSMC proliferation and migration. Thus, miR-503 may be considered a suppressor of postischemic neovascularization in DM and a potential therapeutic target for improving healing of diabetic ischemic tissues (61). In order to identify a specific and sensitive biomarker for ASO, using tissue samples and blood samples obtained from ASO patients, Li et al examined the expression levels of a series of miRNAs. The results identified a significant increase in miR-130a, miR-27b and miR-210 expression, which was positively correlated with the Fontaine stage (59). Therefore, the serum and tissue levels of these miRNAs serve as potential biomarkers for early stage PAD.

\section{Conclusion}

At present, investigating cellular and molecular mechanisms and gene therapy have become hot topics in PAD. Accumulating evidence has demonstrated that multiple miRNAs may serve as novel biomarkers and new therapeutic targets through their important roles in regulating cell proliferation, differentiation and apoptosis. However, these roles should be further investigated in miRNA-based therapy. As a single miRNA is able to regulate a number of target genes, while multiple miRNAs may be regulated by single genes, more studies are required to analyze the complex interactions between specific miRNAs and their targets during PAD. In addition, the detailed cellular and molecular mechanisms of these specific miRNAs should be further investigated to identify ways of preventing and treating PAD.

\section{References}

1. Mughal NA, Russell DA, Ponnambalam S and Homer-Vanniasinkam S: Gene therapy in the treatment of peripheral arterial disease. Br J Surg 99: 6-15, 2012.

2. Thijssen DH, Cable NT and Green DJ: Impact of exercise training on arterial wall thickness in humans. Clin Sci (Lond) 122: 311-322, 2012.

3. Fang J, Song XW, Tian J, Chen HY, Li DF, Wang JF, Ren AJ, Yuan WJ and Lin L: Overexpression of microRNA-378 attenuates ischemia-induced apoptosis by inhibiting caspase-3 expression in cardiac myocytes. Apoptosis 17: 410-423, 2012.

4. Meister G: miRNAs get an early start on translational silencing. Cell 131: 25-28, 2007.

5. Schier AF: The maternal-zygotic transition: death and birth of RNAs. Science 316: 406-407, 2007.

6. Carrington JC and Ambros V: Role of microRNAs in plant and animal development. Science 301: 336-338, 2003.

7. Zampetaki A and Mayr M: MicroRNAs in vascular and metabolic disease. Circ Res 110: 508-522, 2012.

8. Shantikumar S, Caporali A and Emanueli C: Role of microRNAs in diabetes and its cardiovascular complications. Cardiovasc Res 93: 583-593, 2011.

9. Katare R, Riu F, Mitchell K, Gubernator M, Campagnolo P, Cui Y, Fortunato O, Avolio E, Cesselli D, Beltrami AP, Angelini G, Emanueli C and Madeddu P: Transplantation of human pericyte progenitor cells improves the repair of infarcted heart through activation of an angiogenic program involving micro-RNA-132. Circ Res 109: 894-906, 2011.

10. Wang XQ, Nigro P, World C, Fujiwara K, Yan C and Berk BC: Thioredoxin interacting protein promotes endothelial cell inflammation in response to disturbed flow by increasing leukocyte adhesion and repressing kruppel-like factor 2. Circ Res 110: 560-568, 2012.

11. Zakkar M, Luong le A, Chaudhury H, et al: Dexamethasone arterializes venous endothelial cells by inducing mitogenactivated protein kinase phosphatase-1: a novel antiinflammatory treatment for vein grafts? Circulation 123: 524-532, 2011.

12. Pall T, Pink A, Kasak L, Turkina M, Anderson W, Valkna A and Kogerman P: Soluble CD44 interacts with intermediate filament protein vimentin on endothelial cell surface. PLoS One 6: e29305, 2011.

13. Brouillet S, Hoffmann P, Benharouga M, Salomon A, Schaal JP, Feige JJ and Alfaidy N: Molecular characterization of EG-VEGFmediated angiogenesis: differential effects on microvascular and macrovascular endothelial cells. Mol Biol Cell 21: 2832-2843, 2010.

14. Sabatel C, Malvaux L, Bovy N, Deroanne C, Lambert V, Gonzalez ML, Colige A, Rakic JM, Noel A, Martial JA and Struman I: MicroRNA-21 exhibits antiangiogenic function by targeting RhoB expression in endothelial cells. PLoS One 6: e16979, 2011.

15. Parikh VN, Jin RC, Rabello S, et al: MicroRNA-21 integrates pathogenic signaling to control pulmonary hypertension: results of a network bioinformatics approach. Circulation 125: 1520-32, 2012.

16. Zhou J, Wang KC, Wu W, Subramaniam S, Shyy JY, Chiu JJ, Li JY and Chien S: MicroRNA-21 targets peroxisome proliferators-activated receptor-alpha in an autoregulatory loop to modulate flow-induced endothelial inflammation. Proc Natl Acad Sci USA 108: 10355-10360, 2011.

17. Weber M, Baker MB, Moore JP and Searles CD: MiR-21 is induced in endothelial cells by shear stress and modulates apoptosis and eNOS activity. Biochem Biophys Res Commun 393: 643-648, 2010.

18. Harris TA, Yamakuchi M, Ferlito M, Mendell JT and Lowenstein CJ: MicroRNA-126 regulates endothelial expression of vascular cell adhesion molecule 1. Proc Natl Acad Sci USA 105: 1516-1521, 2008.

19. Fish JE, Santoro MM, Morton SU, Yu S, Yeh RF, Wythe JD, Ivey KN, Bruneau BG, Stainier DY and Srivastava D: miR-126 regulates angiogenic signaling and vascular integrity. Dev Cell 15: 272-284, 2008. 
20. Wang S, Aurora AB, Johnson BA, Qi X, McAnally J, Hill JA, Richardson JA, Bassel-Duby R and Olson EN: The endothelial-specific microRNA miR-126 governs vascular integrity and angiogenesis. Dev Cell 15: 261-271, 2008.

21. Zernecke A, Bidzhekov K, Noels H, et al: Delivery of microRNA-126 by apoptotic bodies induces CXCL12-dependent vascular protection. Sci Signal 2: ra81, 2009.

22. Van Solingen C, Seghers L, Bijkerk R, et al: Antagomir-mediated silencing of endothelial cell specific microRNA-126 impairs ischemia-induced angiogenesis. J Cell Mol Med 13: 1577-1585, 2009.

23. Zhu N, Zhang D, Chen S, Liu X, Lin L, Huang X, Guo Z, Liu J, Wang Y, Yuan W and Qin Y: Endothelial enriched microRNAs regulate angiotensin II-induced endothelial inflammation and migration. Atherosclerosis 215: 286-293, 2011.

24. Dentelli P, Rosso A, Orso F, Olgasi C, Taverna D and Brizzi MF: microRNA-222 controls neovascularization by regulating signal transducer and activator of transcription 5A expression. Arterioscler Thromb Vasc Biol 30: 1562-1568, 2010.

25. Fasanaro P, D'Alessandra Y, Di Stefano V, Melchionna R, Romani S, Pompilio G, Capogrossi MC and Martelli F: MicroRNA-210 modulates endothelial cell response to hypoxia and inhibits the receptor tyrosine kinase ligand Ephrin-A3. J Biol Chem 283: 15878-15883, 2008

26. Qin B, Xiao B, Liang D, Xia J, Li Y and Yang H: MicroRNAs expression in ox-LDL treated HUVECs: MiR-365 modulates apoptosis and $\mathrm{Bcl}-2$ expression. Biochem Biophys Res Commun 410: 127-133, 2011.

27. Ghosh G, Subramanian IV, Adhikari N, et al: Hypoxia-induced microRNA-424 expression in human endothelial cells regulates HIF-alpha isoforms and promotes angiogenesis. J Clin Invest 120: 4141-4154, 2010.

28. Ni CW, Qiu H and Jo H: MicroRNA-663 upregulated by oscillatory shear stress plays a role in inflammatory response of endothelial cells. Am J Physiol Heart Circ Physiol 300: 1762-1769, 2011

29. Qin ZX, Yu P, Qian DH, et al: Hydrogen-rich saline prevents neointima formation after carotid balloon injury by suppressing ROS and the TNF-alpha/NF-kappaB pathway. Atherosclerosis 220: 343-350, 2012.

30. Fogelstrand P, Mellander S and Mattsson E: Increased vascular injury reduces the degree of intimal hyperplasia following angioplasty in rabbits. J Vasc Res 48: 307-315, 2011.

31. Davis BN, Hilyard AC, Nguyen PH, Lagna G and Hata A: Induction of microRNA-221 by platelet-derived growth factor signaling is critical for modulation of vascular smooth muscle phenotype. J Biol Chem 284: 3728-3738, 2009.

32. Chen J, Yin H, Jiang Y, Radhakrishnan SK, Huang ZP, Li J, Shi Z, Kilsdonk EP, Gui Y, Wang DZ and Zheng XL: Induction of microRNA-1 by myocardin in smooth muscle cells inhibits cel proliferation. Arterioscler Thromb Vasc Biol 31: 368-375, 2011.

33. Xie C, Huang H, Sun X, Guo Y, Hamblin M, Ritchie RP Garcia-Barrio MT,Zhang J and Chen YE: MicroRNA-1 regulates smooth muscle cell differentiation by repressing Kruppel-like factor 4. Stem Cells Dev 20: 205-210, 2011

34. Cheng Y, Ji R, Yue J, Yang J, Liu X, Chen H, Dean DB and Zhang C: MicroRNAs are aberrantly expressed in hypertrophic heart: do they play a role in cardiac hypertrophy? Am J Pathol 170: 1831-1840, 2007.

35. Ji R, Cheng Y, Yue J, Yang J, Liu X, Chen H, Dean DB and Zhang C: MicroRNA expression signature and antisensemediated depletion reveal an essential role of MicroRNA in vascular neointimal lesion formation. Circ Res 100: 1579-1588, 2007.

36. Lin Y, Liu X, Cheng Y, Yang J, Huo Y and Zhang C: Involvement of MicroRNAs in hydrogen peroxide-mediated gene regulation and cellular injury response in vascular smooth muscle cells. J Biol Chem 284: 7903-7913, 2009.

37. Wang $X$, Cheng Y, Liu X, Yang J, Munoz D and Zhang C: Unexpected pro-injury effect of propofol on vascular smooth muscle cells with increased oxidative stress. Crit Care Med 39: 738-745, 2011

38. Boettger T, Beetz N, Kostin S, Schneider J, Kruger M, Hein L and Braun T: Acquisition of the contractile phenotype by murine arterial smooth muscle cells depends on the Mir143/145 gene cluster. J Clin Invest 119: 2634-2647, 2009.

39. Quintavalle M, Elia L, Condorelli G and Courtneidge SA: MicroRNA control of podosome formation in vascular smooth muscle cells in vivo and in vitro. J Cell Biol 189: 13-22, 2010
40. Xin M, Small EM, Sutherland LB, Qi X, McAnally J, Plato CF, Richardson JA, Bassel-Duby R and Olson EN: MicroRNAs miR-143 and miR-145 modulate cytoskeletal dynamics and responsiveness of smooth muscle cells to injury. Genes Dev 23: 2166-2178, 2009

41. Cordes KR, Sheehy NT, White MP, Berry EC, Morton SU, Muth AN, Lee TH, Miano JM, Ivey KN and Srivastava D: miR-145 and miR-143 regulate smooth muscle cell fate and plasticity. Nature 460: 705-710, 2009.

42. Elia L, Quintavalle M, Zhang J, Contu R, Cossu L, Latronico MV, Peterson KL, Indolfi C, Catalucci D, Chen J, Courtneidge SA and Condorelli G: The knockout of miR-143 and -145 alters smooth muscle cell maintenance and vascular homeostasis in mice: correlates with human disease. Cell Death Differ 16: 1590-1598, 2009.

43. Cheng Y, Liu X, Yang J, Lin Y, Xu DZ, Lu Q, Deitch EA, Huo Y, Delphin ES and Zhang C: MicroRNA-145, a novel smooth muscle cell phenotypic marker and modulator, controls vascular neointimal lesion formation. Circ Res 105: 158-166, 2009.

44. Kishore R, Krishnamurthy P and Losordo DW: Career moves: induced pluripotent cells from human aortic smooth muscle cells can efficiently redifferentiate into parental phenotype. Circ Res 106: 7-9, 2010.

45. Liu X, Cheng Y, Zhang S, Lin Y, Yang J and Zhang C: A necessary role of miR-221 and miR-222 in vascular smooth muscle cell proliferation and neointimal hyperplasia. Circ Res 104: 476-487, 2009.

46. Liu X, Cheng Y, Yang J, Xu L and Zhang C: Cell-specific effects of miR-221/222 in vessels: molecular mechanism and therapeutic application. J Mol Cell Cardiol 52: 245-255, 2012.

47. Chen KC, Wang YS, Hu CY, Chang WC, Liao YC, Dai CY and Juo SH: OxLDL up-regulates microRNA-29b, leading to epigenetic modifications of MMP-2/MMP-9 genes: a novel mechanism for cardiovascular diseases. FASEB J 25: 1718-1728, 2011.

48. Sun SG, Zheng B, Han M, Fang XM, Li HX, Miao SB, Su M, Han Y, Shi HJ and Wen JK: miR-146a and Kruppel-like factor 4 form a feedback loop to participate in vascular smooth muscle cell proliferation. EMBO Rep 12: 56-62, 2011.

49. Yu ML, Wang JF, Wang GK, You XH, Zhao XX, Jing Q and Qin YW: Vascular smooth muscle cell proliferation is influenced by let-7d microRNA and its interaction with KRAS. Circ J 75: 703-709, 2011

50. Zampetaki A, Kiechl S, Drozdov I, Willeit P, Mayr U, Prokopi M, Mayr A, Weger S, Oberhollenzer F, Bonora E, Shah A, Willeit J and Mayr M: Plasma microRNA profiling reveals loss of endothelial miR-126 and other microRNAs in type 2 diabetes. Circ Res 107: 810-817, 2010.

51. Zhang Y, Wang Y, Wang X, Eisner GM, Asico LD, Jose PA and Zeng C: Insulin promotes vascular smooth muscle cell proliferation via microRNA-208-mediated downregulation of $\mathrm{p} 21$. J Hypertens 29: 1560-1568, 2011

52. Su XL, Wang Y, Zhang W, Zhao LM, Li GR and Deng XL: Insulin-mediated upregulation of $\mathrm{K}(\mathrm{Ca}) 3.1$ channels promotes cell migration and proliferation in rat vascular smooth muscle. J Mol Cell Cardiol 51: 51-57, 2011.

53. Li Y, Song YH,LiF, Yang T, Lu YW and Geng YJ: MicroRNA-221 regulates high glucose-induced endothelial dysfunction. Biochem Biophys Res Commun 381: 81-83, 2009.

54. Togliatto G, Trombetta A, Dentelli P, Rosso A and Brizzi MF: MIR221/MIR222-driven post-transcriptional regulation of P27KIP1 and P57KIP2 is crucial for high-glucose- and AGE-mediated vascular cell damage. Diabetologia 54: 1930-1940, 2011

55. Wang XH, Qian RZ, Zhang W, Chen SF, Jin HM and Hu RM: MicroRNA-320 expression in myocardial microvascular endothelial cells and its relationship with insulin-like growth factor-1 in type 2 diabetic rats. Clin Exp Pharmacol Physiol 36: 181-188, 2009.

56. Bonauer A, Carmona G, Iwasaki M, Mione M, Koyanagi M, Fischer A, Burchfield J, Fox H, Doebele C, Ohtani K, Chavakis E, Potente M, Tjwa M, Urbich C, Zeiher AM and Dimmeler S: MicroRNA-92a controls angiogenesis and functional recovery of ischemic tissues in mice. Science 324: 1710-1713, 2009.

57. Grundmann S, Hans FP, Kinniry S, Heinke J, Helbing T, Bluhm F, Sluijter JP, Hoefer I, Pasterkamp G, Bode C and Moser M: MicroRNA-100 regulates neovascularization by suppression of mammalian target of rapamycin in endothelial and vascular smooth muscle cells. Circulation 123: 999-1009, 2011. 
58. Wang M, Li W, Chang GQ, Ye CS, Ou JS, Li XX, Liu Y, Cheang TY, Huang XL and Wang SM: MicroRNA-21 regulates vascular smooth muscle cell function via targeting tropomyosin 1 in arteriosclerosis obliterans of lower extremities. Arterioscler Thromb Vasc Biol 31: 2044-2053, 2011.

59. Li T, Cao H, Zhuang J, Wan J, Guan M, Yu B, Li X and Zhang W: Identification of miR-130a, miR-27b and miR-210 as serum biomarkers for atherosclerosis obliterans. Clin Chim Acta 412: 66-70, 2011.

60. Fichtlscherer S, Zeiher AM and Dimmeler S: Circulating microRNAs: biomarkers or mediators of cardiovascular diseases? Arterioscler Thromb Vasc Biol 31: 2383-2390, 2011.
61. Caporali A, Meloni M, Vollenkle C, Bonci D, Sala-Newby GB, Addis R, Spinetti G, Losa S, Masson R, Baker AH, Agami R, le Sage C, Condorelli G, Madeddu P, Martelli F and Emanueli C: Deregulation of microRNA-503 contributes to diabetes mellitus-induced impairment of endothelial function and reparative angiogenesis after limb ischemia. Circulation 123: 282-291, 2011. 